Open Access Full Text Article

\title{
Emerging Invasive Fungal Infections: Clinical Features and Controversies in Diagnosis and Treatment Processes
}

\author{
Hongliang Zhang' \\ Aiqun Zhu (iD) ${ }^{1,2}$ \\ 'Emergency Department, The Second \\ Xiangya Hospital of Central South \\ University, Changsha, Hunan 4100II, \\ People's Republic of China; ${ }^{2}$ Department \\ of Nursing, The Second Xiangya Hospital \\ of Central South University, Changsha, \\ Hunan 4I00II, People's Republic of \\ China
}

This article was published in the following Dove Press journal: Infection and Drug Resistance

Background: The diagnosis and treatment of invasive fungal infection (IFI) are still challenging due to its complexity and non-specificity. This study was aimed to investigate the clinical features, diagnosis process, and outcomes of patients with emerging IFIs.

Methods: A retrospective review of emerging IFIs in adult patients at a university hospital in China was conducted; diagnoses were based on the criteria of EORTC/MSG 2008.

Results: 145 IFI patients (pulmonary, intestinal and urinary) were enrolled in this study, including 80 proven $(55.2 \%)$, 59 probable $(40.7 \%)$, or 6 possible IFIs $(4.1 \%)$. Among the 126 pulmonary IFIs, the positivity rate for sputum microscopy, sputum culture, and 1.3- $\beta$ D-glucan (BG) test was 54.0\%, 44.4\%, and 37.3\%, respectively. Among the 19 intestinal and urinary IFIs, routine examination of stool or urine and their culture were the main methods of detection. Positive results of 75 detected fungal strains from the samples showed that 30 cases were complicated with one or more bacterial infections. The average length of hospital stay of IFI patients was $14.0(10.0,20.0)$ days. The time from admission to antifungal therapy initiation $(\mathrm{P}<0.001)$, liver cirrhosis $(\mathrm{P}<0.001)$, hematological tumor $(\mathrm{P}<0.001)$, coinfection $(\mathrm{P}=0.019)$ and immune diseases $(\mathrm{P}=0.025)$ were independent predictors of prolonged hospitalization.

Conclusion: Delayed time was the primary predictor of prolonged hospitalization. This prediction is suggested to improve IFI diagnostic and therapeutic process of IFI to promote prognosis.

Keywords: invasive fungal infections, clinical features, microbiological results, risk factors, hospitalization

\section{Introduction}

Within the last century, unprecedented numbers of fungal and fungal-like diseases have led to serious population decreases and extinctions in wild species. Without exception, human beings and their health have been extensively affected. ${ }^{1}$ The incidence of fungal infection has shown an upward trend. ${ }^{2-5}$ Although significant improvements have been made in disease management and treatment of fungal infections, the mortality rate has only increased. ${ }^{6}$ Early diagnosis of invasive fungal infection (IFI) is a difficult in routine clinical practice but key for the initiation of targeted therapies.

The diagnosis of IFI is based on three elements: the clinical examination, imaging results, and confirmation/proof of the causative agent. Lungs are the most common site of fungal infection. Clinical symptoms typically manifest as fever, cough, dyspnea, chest
Department of Nursing, The Second

Xiangya Hospital of Central South

University, No. 139 Renming Middle

Road, Changsha, Hunan 4I00II, People's

Republic of China

$\mathrm{Tel}+8673185292120$

Fax +8673182233525

Email zhuaiqun74@csu.edu.cn 
pain, and hemoptysis; ${ }^{7,8}$ however, these characteristics only present in some patients. Radiologic examination of the lungs of these patients showed various presentations, only a few had typical features of fungal infection, such as denseness, cavitation, an air crescent sign (11\%), or a halo sign $(4.1 \% \sim 7 \%){ }^{8,9}$ some even showed negative results $(15 \%){ }^{10}$ The $1.3-\beta-$ D-glucan (BG) test is used to detect the BG antigen, which is commonly resides on the fungal surface. Therefore, the BG test is considered to be a useful test for "broad-spectrum" fungal marker. Investigations have reported a BG sensitivity of $60.0-87.9 \%$ and specificity of $78.0-80.5 \%{ }^{11-13}$ The Galactomannan (GM) test is aimed at a specific antigen for Aspergillus and is used for the early diagnosis of Aspergillus infection. In a systematic literature review on pediatric invasive aspergillosis, the pooled sensitivity and specificity were reported to be $85 \%$ and $88 \%$, respectively. ${ }^{14}$ Mycological culture from blood, sputum, tracheal secretions, or bronchoalveolar lavage fluid is the preferred method to obtain a definitive diagnosis. However, the overall sensitivity of culture for the diagnosis of IFI is low. ${ }^{15,16}$

The clinical manifestations and imaging features of IFI vary, and their associated hematological and pathological tests are often time-consuming or lacking sensitivity. Therefore, we sought to review the clinical features, diagnosis criteria, and outcomes of emerging IFIs in adult patients in a tertiary hospital setting in China to put forward possible strategies to improve the therapeutic.

\section{Methods}

\section{Setting}

The study was a retrospective review of data from January to December 2018 from a 3000-bed teaching hospital in China. All the case data were obtained from the hospital medical database of the internal medicine department, and all the patients were aged over 14 years. Patients with a history of fungal infection or local fungal infections such as tinea pedis, tinea manus, or skin fungal infections were excluded. Cases were identified using the search term "fungal infection". The information collected included demographic data, underlying diseases, clinical manifestations, radiologic findings, fungal culture of sterile material results (from the peripheral vein or histopathology specimens, bronchoalveolar lavage fluid, swabs, sputum, urine, or stool), direct microscopy test results (bronchoalveolar lavage fluid, swabs, sputum, urine, or stool), serum BG assay results, serum GM assay results, treatments, length of hospital stay and outcome.
The study was approved by the Ethics committee of the Second Xiangya Hospital, China (2016/S014). This study did not need private information involving patient names, ID numbers, home addresses, phone numbers, etc. Therefore, patient consent was not required by the ethics committee. All investigators kept the patient's information strictly to maintain secrecy.

\section{Clinical Definitions}

Cases were categorized as proven, probable or possible IFI according to the revised definitions of the European Organization for Research and Treatment of Cancer/ Mycoses Study Group (EORTC/MSG) 2008. ${ }^{17}$ According to these definitions, proven IFI required the detection of microorganisms in pathological tissues or fungal cultures of sterile materials. Probable IFI was defined as at least one direct microbiological criterion or a positive serum sample. The absence of microorganisms or blood-negative results were considered possible IFIs.

Computed tomography (CT) of the chest, sinus, abdomen or other anatomical sites was the preferred imaging examination. If $\mathrm{CT}$ could not be performed, an X-ray examination was conducted.

ABG turbidimetric analysis (Charles River Company, USA) was used to detect the BG antigen in serum and bronchoalveolar lavage specimens, with a cutoff index of $>50$. An Aspergillus enzyme immunoassay (EIA, the Platellia Aspergillus Ag, Bio-Rad, USA) detected the Aspergillus GM antigen, with a cutoff index of $\geq 0.5$. If the patient had a second BG test or GM test, we recorded the results. Serum specimens were considered positive as long as one of the results was positive.

We also collected the data on the various microscopic fungi species and culture results.

\section{Evaluation of Diagnosis and Treatment}

According to the principles of fungal therapy in China, the use of antifungal medicines is strictly restricted and requires the guidance of an associate professor physician or above. The antifungal medicines include fluconazole, voriconazole, itraconazole, amphotericin, caspofungin, garlicin capsules, and sodium bicarbonate mouthwash. The initial time of microorganism or blood positive results, as well as the initial time of antifungal therapy, were recorded. If the time difference reached three days, the treatment was considered delayed, and the reason was further analyzed. 


\section{Statistical Analysis}

The continuous variables are shown as medians (Q25, Q75) because of their nonnormal distribution in this study. The categorical data were constructed using frequencies and percentages. Categorical variables were compared using the $\chi^{2}$ test on relationship of diagnosis and treatment, and continuous variables compared via the Kruskal-Wallis $H$-test because of its non-normality. General stepwise linear regression models were used to identify associations between the lengths hospital stay and risk factors. $\mathrm{P}<0.05$ was considered statistically significant. The statistical analyses were performed with SPSS version 21.0 (SPSS Inc., Chicago, IL, USA).

\section{Results}

\section{Clinical Characteristics}

There were 145 cases of emerging IFI from January to December 2018. A considerable number of patients underwent treatment in primary hospitals, but the infection did not improve significantly: $70(48.3 \%)$ patients with one month from onset to hospitalization, 48 (33.1\%) patients with two months, $17(11.7 \%)$ patients with three months and $10(6.9 \%)$ patients with over three months. The detailed clinical characteristics are shown in Table 1. The median age of the patients was 63 years (range, 15 to 88 years), and 56 patients (38.6\%) were female. There were 80 patients $(55.2 \%)$ with proven IFI, 59 patients $(40.7 \%)$ with probable IFI, and 6 patients $(4.1 \%)$ with possible IFI. There were 126 pulmonary IFI cases and 19 intestinal or urinary IFI cases. In this study, 130 patients were discharged and 15 died.

\section{Clinical Presentations}

The nonspecific clinical manifestations are shown in Figure 1. Cough $(96,66.2 \%)$, expectoration $(87,60.0 \%)$, fever $(55$, $37.9 \%)$ and dyspnea $(41,28.3 \%)$ were the main clinical manifestations. Other reported symptoms were oral leukoplakia (24, 16.6\%), hemoptysis $(15,10.3 \%)$, chest tightness (14, $9.7 \%)$, chest pain $(6,4.1 \%)$, and edema $(16,11.0 \%)$. Patients with fungal infections of the digestive tract presented gastrointestinal bleeding or diarrhea $(16,11.0 \%)$. Sixteen patients with pulmonary fungal infection had no fever or clinical manifestations in the lungs; they had been hospitalized for other symptoms, such as fatigue and joint pain.

\section{Radiological Findings}

In this study, 6 patients did not undergo an imaging examination, and 16 cases underwent only X-ray examination, as
Table I Demographic and Clinical Characteristics for the Patients with Invasive Fungal Infections(IFI)

\begin{tabular}{|c|c|c|}
\hline Variables & & $n(\%)$ \\
\hline Age[years, M (Q25, Q75)] & & $\begin{array}{l}63.0(51.0, \\
71.0)\end{array}$ \\
\hline Female, n (\%) & & $56(38.6)$ \\
\hline Diagnostic criteria, n (\%) & $\begin{array}{l}\text { Proven IFI } \\
\text { Probable IFI } \\
\text { Possible IFI }\end{array}$ & $\begin{array}{l}80(55.2) \\
59(40.7) \\
6(4.1)\end{array}$ \\
\hline Underlying diseases, $n(\%)$ & $\begin{array}{l}\text { Cardiovascular diseases } \\
\text { Immune diseases } \\
\text { Chronic obstructive } \\
\text { pulmonary disease } \\
\text { Diabetes mellitus } \\
\text { Hematological neoplasms } \\
\text { Gastrointestinal neoplasms } \\
\text { Other tumors } \\
\text { Chronic renal disorder } \\
\text { Tuberculosis } \\
\text { Skin fungi } \\
\text { AIDS } \\
\text { Liver cirrhosis }\end{array}$ & $\begin{array}{l}50(34.5) \\
39(26.9) \\
33(22.8) \\
\\
29(20.0) \\
9(6.2) \\
4(2.8) \\
8(5.5) \\
13(9.0) \\
16(11.0) \\
4(2.8) \\
5(3.4) \\
4(2.8)\end{array}$ \\
\hline $\begin{array}{l}\text { Peripheral vascular access, } \\
\text { n (\%) }\end{array}$ & $\begin{array}{l}\text { Central venous catheter } \\
\text { Hemodialysis tube }\end{array}$ & $\begin{array}{l}3(2.1) \\
6(4.1)\end{array}$ \\
\hline $\begin{array}{l}\text { Mechanical ventilation, } \\
\mathrm{n}(\%)\end{array}$ & & $4(2.8)$ \\
\hline $\begin{array}{l}\text { Catheter/Gastric tube/ } \\
\text { Peritoneal dialysis tube, } \\
\text { n (\%) }\end{array}$ & & $7(4.8)$ \\
\hline $\begin{array}{l}\text { Treatment history (<30 } \\
\text { days), n (\%) }\end{array}$ & $\begin{array}{l}\text { Prednisones } \\
\text { Chemotherapy } \\
\text { Prior antibiotic therapy } \\
\text { ICU }\end{array}$ & $\begin{array}{l}33(22.8) \\
12(8.3) \\
14(9.7) \\
4(2.8)\end{array}$ \\
\hline Long-term bedridden, n (\%) & & $4(2.8)$ \\
\hline Outcome, n (\%) & $\begin{array}{l}\text { Discharged } \\
\text { Death }\end{array}$ & $\begin{array}{l}130(89.7) \\
15(10.3)\end{array}$ \\
\hline
\end{tabular}

shown in Table 2. Of the 126 patients with pulmonary fungal infections, 15 were complicated with intestinal or urethral fungal infections. There were 123 positive casers according to imaging, including 11 inflammatory cases according to X-ray imaging, 90 inflammatory lesions according to CT imaging, 15 pulmonary interstitial lesions according to CT imaging and 7 distinct descriptions (crescent sign, cavitation, patchy nodules).

There were 13 cases of intestinal fungal infection, 4 cases of urethral fungal infection and 2 cases of intestinal 


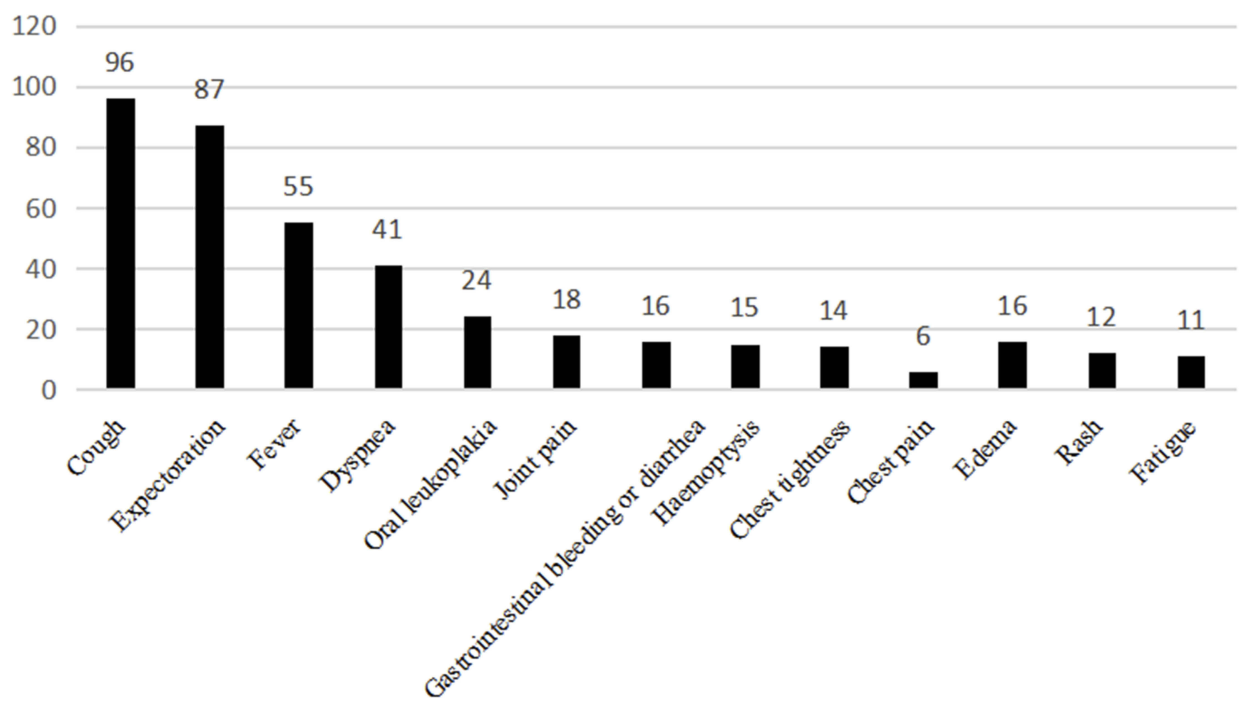

Figure I Clinical manifestations of patients with invasive fungal infection $(n=\mid 45)$.

and urethral infection. Of these patients, 11 showed inflammatory exudation changes on lung imaging, and 1 had typical pulmonary nodules but was negative in sputum microscopy and three cultures.

\section{Microbiological Results} Microbiological Results of Pulmonary IFI

A total of 120 patients met the criteria for proven or probable IFI, and 6 patients met the criteria for possible IFI. As shown in Figure 2, the microbial positivity results were as follows: $18(14.3 \%)$ cases were positive by pharyngeal smear, 68 (54.0\%) cases by sputum microscopy, 56

Table 2 Radiologic Findings of Patients with Invasive Fungal Infections

\begin{tabular}{|c|c|c|}
\hline Radiologic Findings & & $\begin{array}{l}\text { Number } \\
\text { (\%) }\end{array}$ \\
\hline Lung IFI $(n=126)$ & $\begin{array}{l}\text { Not done } \\
\text { Negative } \\
\text { X-ray inflammation } \\
\text { Special description } \\
\text { Pulmonary interstitial } \\
\text { lesions } \\
\text { Inflammatory lesions }\end{array}$ & $\begin{array}{l}2(1.6) \\
1(0.8) \\
11(8.7) \\
7(5.6) \\
15(11.9) \\
90(71.4)\end{array}$ \\
\hline $\begin{array}{l}\text { Intestine or urinary tract IFI } \\
(n=19)\end{array}$ & $\begin{array}{l}\text { Not done } \\
\text { Negative } \\
\text { X-ray inflammation } \\
\text { Special description } \\
\text { Inflammatory lesions }\end{array}$ & $\begin{array}{l}4(21.1) \\
3(15.8) \\
5(26.3) \\
1(5.3) \\
6(31.6)\end{array}$ \\
\hline
\end{tabular}

(44.4\%) cases by sputum culture, 2 cases by microscopic examination of bronchoscopic alveolar lavage fluid (BALF), 3 cases by culture of BALF, 47 (37.3\%) cases by the BG test, 13 cases by the GM test and 5 cases by identification of the cryptococcus antigen.

In clinical practice, routine microbiological examination often requires repeated tests to observe the pathogen. We further analyzed the number of positive first microbiological examinations. The results were as follows: among 68 positive sputum smear microscopy examinations, 52 cases required one examination, 8 cases required two examinations, 8 cases required three or four examinations. Among the 56 positive sputum cultures, 47 cases required one culture, 7 cases required two cultures, and 2 cases required three cultures. Nine patients underwent two $\mathrm{BG}$ tests. Four of the tests changed from negative to positive, four changed from positive to negative, and one did not change. Two GM tests were performed for 11 patients, of which 3 changed from negative to positive and 8 remained unchanged.

Microbiological Results of Intestinal and Urinary IFIs All 19 patients underwent routine stool and urine tests; 12 patients had positive routine stool results and 4 patients had positive routine urine results. Fecal culture was positive in 6 of 8 cases, and urine culture was positive in 2 of 4 cases. Only 2 of 5 cases were positive according to the BG test.

Blood culture was performed in 40 patients in this study; all the results were negative. 


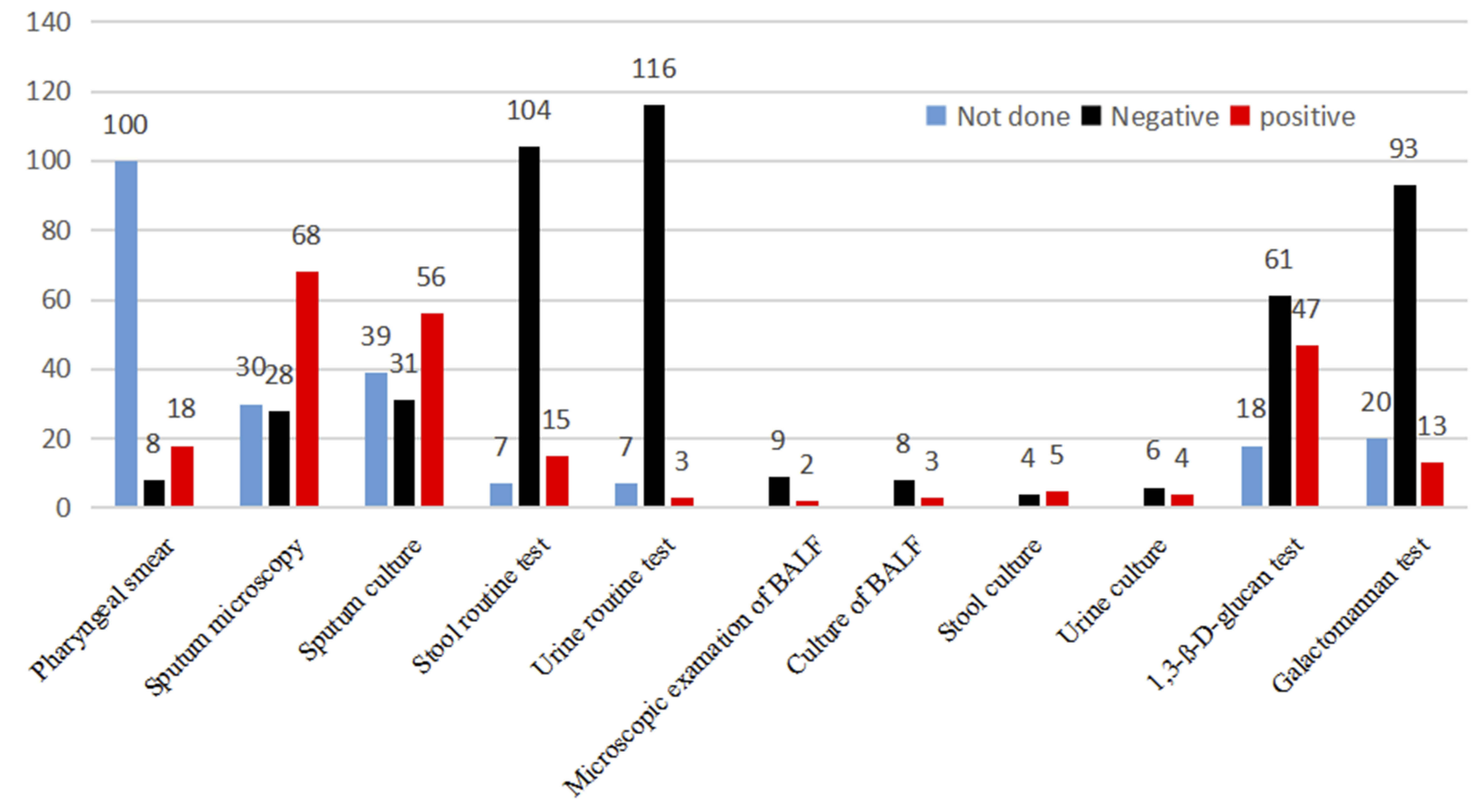

Figure 2 Microbiological results of patients with pulmonary invasive fungal infection $(n=126)$.

\section{Histopathologic Examinations}

3 out of 9 cases were tested positive according to the immunofluorescence assay, 4 of 12 cases were positive according to $\mathrm{A} /$ $\mathrm{P}$ immunohistochemical staining, and 4 of 6 cases were positive according to lung tissue culture. Among them, one case was identified from a duodenal bulb pathological biopsy; fungal hyphae could be seen, and the possibility of Mucor was high.

\section{Fungal Strains}

Based on the results of the various cultures (sputum, stool, and urine) and histopathology, 75 fungal strains were obtained, as shown in Table 3. Fungus (22, 29.3\%), mold

Table 3 Fungal Strains of 75 Patients with Invasive Fungal Infections

\begin{tabular}{|l|l|}
\hline Fungal Strains & Number (\%) \\
\hline Candida albicans & $35(46.7)$ \\
Fungus & $22(29.3)$ \\
Aspergillus fumigatus & $5(6.7)$ \\
Mould & $4(5.3)$ \\
Aspergillus & $3(4.0)$ \\
Candida glabrata & $3(4.0)$ \\
Trichosporon asahii & $2(2.7)$ \\
Saccharomycetes & $\mathrm{I}(1.3)$ \\
\hline
\end{tabular}

(4, 5.3\%), Aspergillus (3, 4.0\%), Aspergillus fumigatus (5, 6.7\%), Candida albicans $(35,46.7 \%)$, Saccharomycetes (1, $1.3 \%)$, Candida glabrata $(3,4.0 \%)$, and Trichosporon asahii $(2,2.7 \%)$ were identified. The culture results showed that 30 patients were complicated with one or more bacterial infections, including Klebsiella pneumoniae $(\mathrm{n}=10)$, grampositive bacteria $(n=9)$, gram-negative bacteria $(n=8)$, Pseudomonas aeruginosa $(\mathrm{n}=4)$, Serratia marcescens $(n=2)$, Acinetobacter baumannii $(n=2)$ and Penicillium martensii $(\mathrm{n}=1)$.

\section{Diagnosis and Treatment}

13 of 145 patients did not receive antifungal therapy during hospitalization. 7 of these patients had no diagnosis of fungal infection in their discharge records. Although there were slight differences in the medical treatments $(\mathrm{p}=0.020)$, the length of hospital stay $(p=0.038)$ and the time from admission to the initiation of antifungal therapy ( $\mathrm{p}=0.032$ ) among the three groups (proven IFI, probable IFI, and possible IFI), there were no statistically significant differences in the outcomes (discharge or death), as shown in Table 4. In the overall sample, the average length of hospital stay was 14.0 $(10.0,20.0)$ days, the average time from admission to the acquisition of positive etiological or hematological results was $2.0(1.0,4.5)$ days, and the average time 
Table 4 Results of Diagnosis and Treatment of Patients with Invasive Fungal Infections

\begin{tabular}{|c|c|c|c|c|c|}
\hline Variables & $\begin{array}{l}\text { Proven IFI } \\
(n=80)\end{array}$ & $\begin{array}{l}\text { Probable IFI } \\
(n=59)\end{array}$ & $\begin{array}{l}\text { Possible IFI } \\
(n=6)\end{array}$ & $\chi^{2}$ & $\mathbf{P}$ \\
\hline \multicolumn{6}{|l|}{ Discharge diagnosis, $\mathrm{n}(\%)$} \\
\hline Yes & $78(97.5)$ & $54(91.5)$ & $6(100.0)$ & 2.96 & 0.228 \\
\hline No & $2(2.5)$ & $5(8.5)$ & 0 & & \\
\hline \multicolumn{6}{|l|}{ Antifungal therapy, $n(\%)$} \\
\hline Yes & $77(96.3)$ & $49(83.0)$ & $6(100.0)$ & 7.87 & 0.020 \\
\hline No & $3(3.7)$ & $10(17.0)$ & 0 & & \\
\hline Length of hospital stay, [days, M (Q25, Q75)] & $16.0(10.3,25.8)$ & $12.0(9.0,18.0)$ & $13.0(13.0,17.0)$ & 6.53 & 0.038 \\
\hline $\begin{array}{l}\text { The time from admission to the initiation of antifungal therapy, } \\
\text { [days, } M(Q 25, Q 75)]\end{array}$ & $7.0(3.0,12.0)$ & $4.0(1.0,8.0)$ & $4.0(1.0,7.0))$ & 6.87 & 0.032 \\
\hline $\begin{array}{l}\text { The time from admission to the acquisition of positive etiological or } \\
\text { hematological results, [days, } M(Q 25, Q 75) \text { ] } \\
\text { Outcomes, } n(\%)\end{array}$ & $3.0(1.0,6.0)$ & $1.0(1.0,3.0)$ & - & 9.10 & 0.003 \\
\hline Discharge & $70(87.5)$ & $54(91.5)$ & $6(100.0)$ & 1.32 & 0.518 \\
\hline Death & $10(12.5)$ & $5(8.5)$ & 0 & & \\
\hline
\end{tabular}

from admission to the initiation of antifungal therapy was $6.0(2.0,11.0)$ days. Delayed antifungal therapy was reported in $54(37.2 \%)$ patients. The main reasons included waiting for the second diagnosis of the pathogen $(n=35)$, waiting for consultation $(n=4)$, waiting for senior physician rounds $(n=9)$, considering specimens contamination $(n=2)$ and 3 cases received medication at discharge.

\section{Regression Analysis of Hospitalization}

\section{Time}

The logistic regression analysis showed that hospitalization time was closely associated with the time from admission to antifungal therapy initiation $(\mathrm{P}<0.001)$, liver cirrhosis $(\mathrm{P}<0.001)$, hematological tumor presence $(\mathrm{P}<0.001)$, coinfection $(\mathrm{P}=0.019)$ and immune diseases $(\mathrm{P}=0.025)$, as shown in Table 5.

\section{Discussion}

Invasive mycosis is a significant cause of mortality, but its diagnosis is still a great challenge due of its atypical clinical features and uncertain laboratory test results; these factors directly affect its treatment and prognosis. Invasive fungal infections can occur in any part of the body and sometimes involve multiple organs or tissues, with the lungs being the most common. The common risk factors we observed, such malignant tumors, immune diseases, chronic obstructive pulmonary disease and so on, were similar to those in previous reports, but cardiovascular diseases and diabetes mellitus were also major influential diseases in this study. People with diabetes mellitus are

Table 5 Regression Analysis of Hospitalization Time and Related Factors

\begin{tabular}{|c|c|c|c|c|c|c|c|}
\hline & B & S.E. & Beta & $\mathbf{t}$ & Sig. & $95 \% \mathrm{Cl}$ & \\
\hline Constant & 7.94 & 1.26 & & 6.31 & $<0.001$ & 5.45 & 10.43 \\
\hline The time from admission to the initiation of antifungal therapy & 0.85 & 0.12 & 0.46 & 6.86 & $<0.001$ & 0.60 & 1.09 \\
\hline Liver cirrhosis & 16.59 & 4.20 & 0.27 & 3.95 & $<0.001$ & 8.28 & 24.89 \\
\hline Hematological tumors & 10.58 & 2.83 & 0.25 & 3.74 & $<0.001$ & 4.97 & 16.18 \\
\hline Co-infection & 4.03 & 1.69 & 0.16 & 2.38 & 0.019 & 0.68 & 7.38 \\
\hline Immune diseases & 3.51 & 1.55 & 0.15 & 2.26 & 0.025 & 0.44 & 6.58 \\
\hline
\end{tabular}

Notes: In stepwise linear regression analysis, hospitalization time is the dependent variable. $\mathrm{B}=$ unstandardized regression coefficient, Beta $=$ standardized coefficient. Independent variables are gender, age, diabetes mellitus, hematological neoplasms, gastrointestinal neoplasms, other tumors, cardiovascular diseases, chronic obstructive pulmonary disease, chronic renal disorder, immune diseases, liver cirrhosis, tuberculosis, acquired immune deficiency syndrome, intensive care unit, mechanical ventilation, prednisones, chemotherapy, peripheral vascular access, catheter/gastric tube/peritoneal dialysis tube, co-infection, delayed antifungal therapy, the time from admission to the acquisition of positive etiological or hematological results, the time from admission to the initiation of antifungal therapy.

Abbreviation: SE, standard error. 
more susceptible to infection, especially fungal infection, as a result of neutrophil dysfunction and impaired humoral immunity. $^{18,19}$

The clinical manifestations of the patients were atypical. Fever, which is a common infection symptom, presented in only $37.9 \%$ of the patients. There were no pulmonary symptoms or signs in several pulmonary IFI patients, and 2 patients in this study did not undergo any radiological examinations. Although imaging findings associated with fungal infections are typically characterized by halo signs, air crescent signs and nodules, the results in this study were nonspecific. The most common manifestations were inflammatory lesions and pulmonary interstitial lesions. The halo sign and air crescent sign were observed in only $8(5.5 \%)$ patients, which was significantly lower than the $40.0 \%^{20}$ or $62.5 \%^{21}$ of patients reported in other studies It is possible that only 8 aspergillosis cases were confirmed by microbiological examination as the air crescent sign is a late sign of invasive aspergillosis. ${ }^{22}$ Some studies emphasized the transient nature of the halo sign; its incidence was particularly high during the early stages, but over time, the halo sign tended to disappear. ${ }^{23,24} \mathrm{CT}$ pulmonary interstitial lesions are often associated with autoimmune diseases. In the diagnosis of these patients, we should consider whether the patients are complicated with fungal infections.

According to IFI guidelines, a definitive diagnosis of proved IFI depends on the microbiological analysis or culture of sterile material, such as sputum results. Despite repeated tests, the positive rate of sputum culture was only $44.4 \%$. The positivity rate of microbial detection in bronchoalveolar lavage fluid was not high, and only 3 of 11 patients were positive. The detection of pathological tissues is often used to obtain a diagnosis when no definite microorganisms can be found in the body fluids. In this study, the positivity rate of microorganisms in 12 pathological tissue samples was nearly one-third. A definitive diagnosis of probable IFI relies on direct testing (cytology, direct microscopy, or culture) or indirect testing (detection of antigen or cell-wall constituents). The positivity rate according to sputum microscopy increased slightly after the repeated collection of specimens. Among the cases of pulmonary fungal infection, the positivity rate according to sputum microscopy was 68 $(54.0 \%)$, followed by the BD test at $47(37.3 \%)$. The positivity rate of the $\mathrm{BD}$ test was significantly lower than $60 \%$, which has been reported in some studies. ${ }^{13,25}$ This result is probably because, in $51.7 \%$ of the patients in this study, the time from disease onset to hospitalization was 1 to 3 months, similar to Angebault's study, ${ }^{25}$ which showed that BG negativity occurred at $>1$ month for candidemia and $>3$ months for invasive aspergillosis. In cases of intestinal and urinary tract fungal infections, the positivity rates of routine stool, routine urine and culture tests were significantly increased. To obtain a definitive diagnosis, direct microscopy and culture are economical, rapid and effective methods, despite the need for multiple specimens.

Among the 75 positive results based on culture and pathology, Candida albicans was the main pathogenic agent, followed by fungi and mold. This is consistent with Li's research from China. ${ }^{7}$ In this study, 30 cases of IFI were complicated with one or more bacterial infection, which posed a challenge in the diagnosis of the fungal infections, as the symptoms and imaging findings were more complex.

The diagnosis and treatment of IFI are very complicated. Before administering antifungal treatments, we hope to find microbiological evidence for a definitive diagnosis. In this study, 54 out of 139 proven or probable IFIs were not treated until secondary etiological results or confirmation from a superior physicians was obtained. If patients present IFIrelated clinical symptoms or risk factors and direct or indirect positive microbiological results, must they wait for a second positive result before receiving antifungal treatment? In fact, the positivity rate of direct or indirect microorganism detection by a single test is quite low. In this process, it takes an average of an additional four days to initiate antifungal treatment. Obviously, the length of stay was also extended by four days in Table 4. There were still some patients who were not definitively diagnosed with fungal infections or did not receive antifungal therapy until discharge. However, all six possible IFI patients were diagnosed and treated. The possible reason was that those who did not use drugs had mild cases, and the fungal infections were controlled while the underlying diseases improved and the body's immunity strengthened. ${ }^{26,27}$ In addition to liver cirrhosis, hematological tumor, coinfection and immune diseases, the time from admission to the initiation of antifungal therapy was related to the prolongation of hospitalization. The mean hospital LOS of $14.0(10.0,20.0)$ days was similar to those ranging from 14 days to 16 days in one studies ${ }^{28}$ and significantly lower than those ranging from 32.4 to 36 days in other studies. $^{29,30}$ It may be that patients in the former hospital were discharged after they had achieved the discharge standard and continued to take the medicine at home until a sufficient course of treatment was achieved. Automated ID consultations can improve fungal-related clinical issues but may not impact the time to appropriate antifungal 
therapy. ${ }^{28}$ Medical experts unanimously agreed that microscopy and culture were the basic diagnostic tests for fungal infection at present, ${ }^{31,32}$ and this study suggested that the consultations by superior physicians should be simple and timely so that patients can receive antifungal treatment as soon as possible to reduce hospital stays and medical expenses.

\section{Conclusions}

Because of atypical clinical manifestations, low incidence of specific signs in imaging examinations and low sensitivity in microbial detection, IFI is diagnosed and treated with antifungal therapy often after multiple tests and consultations with physicians. In addition to its underlying disease and co-infection, this study emphasized that the time from admission to the initiation of antifungal treatment was associated with the length of hospitalization. This result suggests that we need to improve IFI diagnostic and therapeutic criteria and optimize management procedures so that patients with fungal infections can receive treatment as soon as possible to improve prognoses.

\section{Ethics Approval}

Ethics approval was obtained from the Ethics committee of the Second Xiangya Hospital, China (2016/S014). This retrospective study did not need private information involving patient names, ID numbers, home addresses, phone numbers, etc. Therefore, patient or parental consent was not required by the ethics committee. All investigators kept the patient's information strictly to maintain secrecy and abided by the declaration of Helsinki.

\section{Acknowledgments}

The authors thank the hospital for its support, especially the medical record information department. We also thank the Emergency Medical Team for its recommendations.

\section{Author Contributions}

A.Z designed this survey, H.Z and A.Z collected data. All authors contributed to data analysis, drafting or revising the article, gave final approval of the version to be published, and agree to be accountable for all aspects of the work.

\section{Funding}

This study was supported by grants from the Science and Technology Project of Hunan Province, China (grant number: 2015SK2028).

\section{Disclosure}

The authors declare that they have no competing interests in this work.

\section{References}

1. Fisher MC, Henk DA, Briggs CJ, et al. Emerging fungal threats to animal, plant and ecosystem health. Nature. 2012;484:186-194. doi:10.1038/nature 10947

2. Cho SH, Yu YB, Park JS, Yook KD, Kim YK. Epidemiological characterization of imported systemic mycoses occurred in Korea. Osong Public Health Res Perspect. 2018;9:255-260. doi:10.24171/j. phrp.2018.9.5.07

3. Tarka P, Nitsch-Osuch A, Gorynski P, Tyszko P, Bogdan M, Kanecki K. Epidemiology of pulmonary aspergillosis in hospitalized patients in poland during 2009-2016. Adv Exp Med Biol. 2019;1160:73-80.

4. Hoffmann-Santos HD, Paula CR, Yamamoto AC, Tadano T, Hahn RC. Six-year trend analysis of nosocomial candidemia and risk factors in two intensive care hospitals in Mato Grosso, midwest region of Brazil. Mycopathologia. 2013;176:409-415. doi:10.1007/ s11046-013-9705-5

5. Webb BJ, Ferraro JP, Rea S, Kaufusi S, Goodman BE, Spalding J. Epidemiology and clinical features of invasive fungal infection in a US health care network. Open Forum Infect Dis. 2018;5:ofy187. doi:10.1093/ofid/ofy 187

6. Dounia B, Olivier L, Yann LS, et al. Population-based analysis of invasive fungal infections, France, 2001-2010. Emerg Infect Dis. 2014;20:1149-1155. doi:10.3201/eid2007.140087

7. Peng L, Xu Z, Huo Z, Long R, Ma L. New insights into the clinical characteristics and prognostic factors of pulmonary fungal infections from a retrospective study in Southwestern China. Infect Drug Resist. 2018;11:307-315. doi:10.2147/IDR

8. Rotjanapan P, Chen YC, Chakrabarti A, et al. Epidemiology and clinical characteristics of invasive mould infections: a multicenter, retrospective analysis in five Asian countries. Med Mycol. 2018;56:186-196. doi:10.1093/mmy/myx029

9. Anugulruengkitt S, Trinavarat P, Chantranuwat P, Sritippayawan S, Pancharoen C, Thanyawee P. Clinical features and survival outcomes of invasive aspergillosis in pediatric patients at a medical school in Thailand. J Med Assoc Thai. 2016;99:150-158.

10. Fracchiolla NS, Sciumè $M$, Orofino N, et al. Epidemiology and treatment approaches in management of invasive fungal infections in hematological malignancies: results from a single-centre study. PLoS One. 2019;14:e0216715. doi:10.1371/journal.pone.0216715

11. Dichtl K, Seybold U, Wagener J. Serological biomarkers of candidemia: a retrospective evaluation of three assays. Infection. 2019;47:217-224. doi:10.1007/s15010-018-1224-3

12. Pini P, Bettua $C$, Orsi CF, et al. Evaluation of serum $(1 \rightarrow 3)-\beta$ D-glucan clinical performance: kinetic assessment, comparison with galactomannan and evaluation of confounding factors. Infection. 2016;44:223-233. doi:10.1007/s15010-015-0849-8

13. Furfaro E, Giacobbe DR, Del Bono V, et al. Performance of serum (1,3)-B-d-glucan screening for the diagnosis of invasive aspergillosis in neutropenic patients with haematological malignancies. Mycoses. 2018;61:650-655. doi:10.1111/myc.2018.61.issue-9

14. Tong T, Shen J, Xu Y. Serum galactomannan for diagnosing invasive aspergillosis in pediatric patients: a meta-analysis. Microb Pathog. 2018;118:347-356. doi:10.1016/j.micpath.2018.03.059

15. van Paassen J, Russcher A, In 't Veld-van Wingerden AW, Verweij PE, Kuijper EJ. Emerging aspergillosis by azole-resistant aspergillus fumigatus at an intensive care unit in the Netherlands, 2010 to 2013. Euro Surveill. 2016;21:1-9. doi:10.2807/1560-7917. ES.2016.21.30.30300 
16. Ruhnke M, Schwartz S. Recent developments in the management of invasive fungal infections in patients with oncohematological diseases. Ther Adv Hematol. 2016;7:345-359. doi:10.1177/ 2040620716656381

17. De Pauw B, Walsh TJ, Donnelly JP, et al. Revised definitions of invasive fungal disease from the European Organization for Researchand Treatment of Cancer/Invasive Fungal Infections Cooperative Group and the National Institute of Allergy and Infectious Diseases Mycoses Study Group (EORTC/MSG) Consensus Group. Clin Infect Dis. 2008;46:1813-1821. doi:10.1086/588660

18. Rodrigues CF, Rodrigues ME, Henriques M, Candida SP. Infections in patients with diabetes mellitus. J Clin Med. 2019;8:1-41. doi: $10.3390 / \mathrm{jcm} 8010076$

19. Cohen Atsmoni S, Brener A, Roth Y. Diabetes in the practice of otolaryngology. Diabetes Metab Syndr. 2019;13:1141-1150. doi:10.1016/j.dsx.2019.01.006

20. Rieger H, Lustig D, Barlow S, et al. Applicability of the EORTC/ MSG criteria for IFD in clinical practice. Ann Hematol. 2015;94:847-855. doi:10.1007/s00277-014-2282-y

21. Nucci F, Nouér SA, Capone D, Nucci M. Invasive mould disease in haematologic patients: comparison between fusariosis and aspergillosis. Clin Microbiol Infect. 2018;24:1105.e1-1105.e4. doi:10.1016/j.cmi.2018.05.006

22. Georgiadou SP, Sipsas NV, Marom EM, Kontoyiannis DP. The diagnostic value of halo and reversed halo signs for invasive mold infections in compromised hosts. Clin Infect Dis. 2011;52:1144-1155. doi: $10.1093 / \mathrm{cid} / \mathrm{cir} 122$

23. Brook O, Guralnik L, Hardak E, et al. Radiological findings of early invasive pulmonary aspergillosis in immune-compromised patients. Hematol Oncol. 2009;27:102-106. doi:10.1002/hon.v27:2

24. Brodoefel H, Vogel M, Hebart H, et al. Long-term CT follow-up in 40 non-HIV immunocompromised patients with invasive pulmonary aspergillosis: kinetics of CT morphology and correlation with clinical findings and outcome. AJR Am J Roentgenol. 2006;187:404-413. doi:10.2214/AJR.05.0513
25. Angebault C, Lanternier F, Dalle F, et al. Prospective evaluation of serum $\beta$-glucan testing in patients with probable or proven fungal diseases. Open Forum Infect Dis. 2016;3:ofw128. doi:10.1093/ofid/ ofw128

26. Shourian M, Qureshi ST. Resistance and tolerance to cryptococcal infection: an intricate balance that controls the development of disease. Front Immunol. 2019;10:66. doi:10.3389/fimmu.2019.00066

27. Kobayashi S, Soyama A, Takatsuki M, et al. Relationship between immune function recovery and infectious complications in patients following living donor liver transplantation. Hepatol Res. 2016;46:908-915. doi:10.1111/hepr.v46.9

28. Jones TM, Drew RH, Wilson DT, Sarubbi C, Anderson DJ. Impact of automatic infectious diseases consultation on the management of fungemia at a large academic medical center. Am J Health Syst Pharm. 2017;74:1997-2003. doi:10.2146/ajhp170113

29. Moghnieh R, Alothman AF, Althaqafi AO, et al. Epidemiology and outcome of invasive fungal infections and methicillin-resistant staphylococcus aureus (MRSA) pneumonia and complicated skin and soft tissue infections(cSSTI) in Lebanon and Saudi Arabia. J Infect Public Health. 2017;10:849-854. doi:10.1016/j.jiph.2017.01.013

30. Aljeboori Z, Gorelik A, Jenkins E, McFarlane T, Darvall J. Risk factors for candidaemia and their cumulative effect over time in a cohort of critically ill, non-neutropenic patients. Crit Care Resusc. 2018;20:313-319.

31. Hay R, Denning DW, Bonifaz A, et al. The diagnosis of fungal neglected tropical diseases (fungal NTDs) and the role of investigation and laboratory tests: an expert consensus report. Trop Med Infect Dis. 2019;4:E122. doi:10.3390/tropicalmed4040122

32. Cornely OA, Alastruey-Izquierdo A, Arenz D, et al. Global guideline for the diagnosis and management of mucormycosis: an initiative of the European confederation of medical mycology in cooperation with the Mycoses Study Group Education and Research Consortium. Lancet Infect Dis. 2019;19:e405-e421. doi:10.1016/S1473-3099(19) 30312-3
Infection and Drug Resistance

\section{Publish your work in this journal}

Infection and Drug Resistance is an international, peer-reviewed openaccess journal that focuses on the optimal treatment of infection (bacterial, fungal and viral) and the development and institution of preventive strategies to minimize the development and spread of resistance. The journal is specifically concerned with the epidemiology of
Dovepress

antibiotic resistance and the mechanisms of resistance development and diffusion in both hospitals and the community. The manuscript management system is completely online and includes a very quick and fair peerreview system, which is all easy to use. Visit http://www.dovepress.com/ testimonials.php to read real quotes from published authors 